\title{
BMJ Open Trends in prescribing pattern of opioid and benzodiazepine substitutes among Medicare part D beneficiaries from 2013 to 2018: a retrospective study
}

\author{
Aimalohi Esechie, ${ }^{1}$ Yong-Fang Kuo, ${ }^{2}$ James S Goodwin, ${ }^{3}$ Jordan Westra, ${ }^{4}$ \\ Mukaila A Raji (i) ${ }^{5}$
}

To cite: Esechie A, Kuo Y-F, Goodwin JS, et al. Trends in prescribing pattern of opioid and benzodiazepine substitutes among Medicare part D beneficiaries from 2013 to 2018 : a retrospective study. BMJ Open 2021;11:e053487. doi:10.1136/ bmjopen-2021-053487

- Prepublication history and additional supplemental material for this paper are available online. To view these files, please visit the journal online (http://dx.doi.org/10.1136/ bmjopen-2021-053487).

Received 21 May 2021 Accepted 25 October 2021

Check for updates

(C) Author(s) (or their employer(s)) 2021. Re-use permitted under CC BY-NC. No commercial re-use. See rights and permissions. Published by BMJ.

For numbered affiliations see end of article.

Correspondence to

Dr Mukaila A Raji;

muraj@utmb.edu

\section{ABSTRACT}

Objective Opioid and benzodiazepine co-prescribing is associated with a substantial increase in opioid overdose deaths. In this study, we examine the prescribing trends of substitutes of opioids and benzodiazepines alone or in combination, compared with opioids and benzodiazepines. Design Retrospective cohort study.

Setting Data were collected using a $20 \%$ national sample of Medicare beneficiaries from 2013 to 2018.

Participants 4.1-4.3 million enrollees each year from 2013 to 2018.

Intervention None.

Primary outcome We employ a generalised linear mixed models to calculate $\mathrm{ORs}$ for opioid use, benzodiazepine or Z-drug (benzos/Z-drugs) use, opioid/benzos/Z-drugs 30-day use, gabapentinoid use and (selective serotonin reuptake inhibitors (SSRI) and serotonin norepinephrine reuptake inhibitors (SNRIs)) use, adjusted for the repeated measure of patient. We then created two models to calculate the ORs for each year and comparing to 2013. Results Opioid and benzos/Z-drugs use decreased by 2018 (aOR 0.626; 95\% Cl 0.622 to 0.630) comparing to 2013 . We demonstrate a $36.3 \%$ and $9.9 \%$ increase rate of gabapentinoid and SSRI/SNRI use, respectively. Furthermore, combined gabapentinoid and SSRI/SNRI use increased in 2018 (aOR 1.422; 95\% Cl 1.412 to 1.431). Conclusion Little is known about the prescribing pattern and trend of opioid and benzodiazepine alternatives as analgesics. There is a modest shift from prescribing opioid and benzos/Z-drugs (alone or in combination) towards prescribing non-opioid analgesics — gabapentinoids with and without non-benzos/Z-drugs that are indicated for anxiety. It is unclear if this trend towards opioid/benzos/Zdrugs alternatives is associated with fewer drug overdose death, better control of pain and comorbid anxiety, and improved quality of life.

\section{INTRODUCTION}

Opioid use contributes to over two-thirds of drug overdose-related deaths in the USA. ${ }^{1}$ This finding prompted the US Drug Enforcement Administration, ${ }^{2}$ the Centers for Disease Control and Prevention (CDC), ${ }^{3}$ state governments and health payers to implement policies and guidelines limiting prescriptions
Strengths and limitations of this study

- This is a retrospective study.

- Our study uses large Medicare data set to analyse the effect of the 2016, the Centers for Disease Control and Prevention (CDC) published guidelines for all clinicians to 'avoid concurrent opioid and benzodiazepine prescribing' in response to the growing opioid overdose death epidemic.

- Multilevel and longitudinal analysis used in this study identified patterns in prescribing study outcomes.

- Analysis of year as a categorical and dichotomised variable allowed for identification of the effect of 2016 CDC guidelines.

- The limitation of this study is that the data represent individuals who filled prescriptions.

of opioid analgesics, especially for noncancer pain..$^{2-4}$ Following the publication of these guidelines, there has been a decrease in the overall number of opioid prescriptions, including a 54\% decline in initial opioid prescription between 2012 and 2017..$^{5-9}$

Risks of opioid overdose and related hospitalisations are increased with concurrent use of benzodiazepines and other central nervous system depressants. ${ }^{10-12}$ Both drug classes can be sedating, suppress respiratory efforts, impair thought, slow response time and increase falls. Several studies described the trend in concomitant use of benzodiazepines with prescription opioids prior to the 2016 CDC guidelines. ${ }^{13-15}$ For example, Sun et $a l^{13}$ described an increase in the rate of co-prescribed opioids and benzodiazepine in privately insured enrollees, from $9 \%$ in 2001 to $17 \%$ in 2013 . This trend in co-prescribing came with a substantial increase in the risk of both fatal and nonfatal opioid overdose. ${ }^{13-15}$ In 2016, the CDC published a guideline for all clinicians to 'avoid concurrent opioid and benzodiazepine prescribing,. ${ }^{3}$ Following the 
CDC guideline, several studies have reported a decrease in opioid and benzodiazepine co-prescribing. ${ }^{15-17}$ For example, Wang et $a l^{18}$ reported more than $15 \%$ relative reduction in the number of recipients with opioidbenzodiazepines overlapping prescription from 2012 to 2017. ${ }^{17}$

In parallel with decline in opioid use, gabapentinoids are increasingly being prescribed by clinicians as a potential alternative to opioid analgesics, with gabapentinoids prescribing rate rising since the 2016 CDC opioidprescribing guideline for chronic pain. ${ }^{18-20}$ Patients with chronic pain have a high rate of co-occurrence of anxiety disorders for which benzodiazepines are frequently prescribed, a prescribing practice associated with high risk of opioid overdose death. ${ }^{10-15}{ }^{21-23}$ While the prevalence of anxiety disorders vary according to the sources of chronic pain-back pain versus abdominal pain, McWilliams $e t a l^{23}$ showed a $35.1 \%$ prevalence of anxiety disorders among the community-dwelling participants of the US National Comorbidity Survey who reported chronic pain, a rate of almost twice that of anxiety disorder prevalence $(18.1 \%)$ in the control general population. It is unclear the extent to which clinicians have shifted from benzodiazepine prescribing to guideline-recommended serotonergic anxiolytics as alternative drug for the comorbid anxiety disorders in chronic pain patients. The most common serotonergic anxiolytics are the selective serotonin reuptake inhibitors (SSRI) and serotonin norepinephrine reuptake inhibitors (SNRI), and because of their safety profile even in overdose, they are possible substitutes for benzodiazepines in patients with co-occurring anxiety and pain. ${ }^{24-27}$

There are no studies on whether the use of potential substitute drugs (gabapentinoids and SSRIs/SNRIs) increased as opioids and benzodiazepines decreased. We hypothesised that opioid and benzodiazepine prescriptions would decrease while substitute medications, gabapentinoids and SSRIs/SNRIs, would increase in

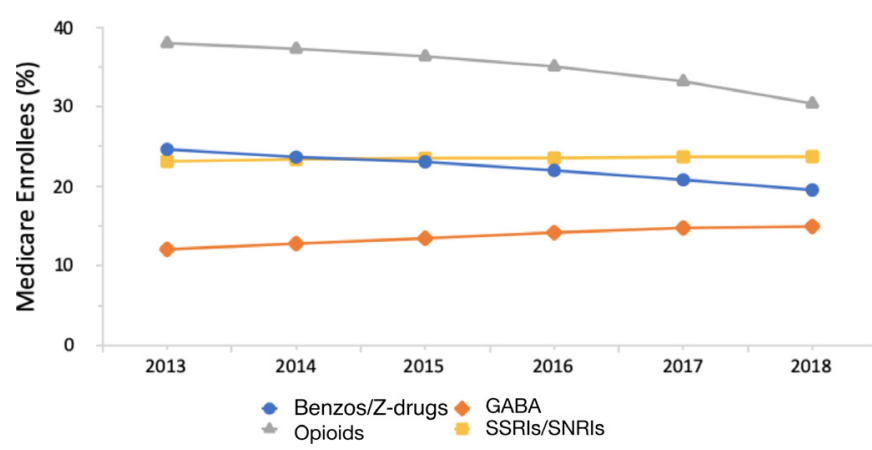

Figure 1 The change over time in the percentage of Medicare enrollees who received a prescription for either opioid, benzos/Z-drugs, GABA or selective serotonin reuptake inhibitors/serotonin norepinephrine reuptake inhibitors (SSRIs/SNRIs) from 2013 to 2018. Decrease in opioid and benzos/Z-drugs prescriptions occurred throughout the study period, and GABA and SSRIs/SNRIs prescription increased. GABA, gabapentinoids (pregabalin and gabapentin). response to the published and widely disseminated 2016 CDC opioid and benzodiazepine guidelines. We thus examined the correlates and time trends-from 2013 to 2018-in prescribing of opioids, benzodiazepines, gabapentinoids and SSRIs/SNRIs alone and in combinations among Medicare beneficiaries, before and after the 2016 CDC guideline.

\section{METHODS}

\section{Source of data}

Data were collected using a 20\% national sample of Medicare beneficiaries from 2013 to 2018, from which cohorts for each year were created. Beneficiaries were excluded from a yearly cohort if they did not have Medicare parts D coverage for the entire calendar year or until death. We further excluded beneficiaries who did not have part A and B coverage or have HMO coverage that year or in the prior year (see online supplemental etable 1; online supplemental results).

\section{Study outcomes}

The outcomes were prescriptions for opioids, benzodiazepines or Z-drugs, gabapentinoids or SSRIs/SNRIs during the calendar year. Consistent with past research, ${ }^{28}{ }^{29}$ we chose to combine Z-drugs and benzodiazepines because of their shared actions as benzodiazepine receptor agonists ${ }^{28-30}$ with overlapping side effect profiles. While we recognise that some pharmacodynamic/kinetic differences exist among the constituent drugs comprising benzodiazepine receptor agonist drug group (eg, chlordiazepoxide vs alprazolam; zolpidem vs zaleplon) and the SSRIs/SNRIs drug group (fluoxetine vs paroxetine vs duloxetine), benzodiazepines and Z-drugs are henceforth referred to as benzos/Z-drugs while the serotonergic agents are referred to as SSRIs/SNRIs, a reflection of shared mechanisms and toxicity profile by drugs in each group. Gabapentinoids comprise pregabalin and gabapentin and are henceforth referred to as GABA. We calculated the proportion of the population in each year that had any filled prescription of each of the drugs alone, as well as the following combinations: opioids and benzos/Z-drugs, opioids and SSRIs/SNRIs, GABA and benzos/Z-drugs, GABA and SSRIs/SNRIs. We also calculated the proportion of the population with 30-day combination prescriptions of opioids and benzos/Z-drugs or GABA and benzos/Z-drugs.

\section{Study measures}

The main independent variable was year, first with each year considered as a categorical variable and then as a dichotomised variable (2013/2014 vs 2017/2018 representing years before and after the publication of the CDC guideline). Other variables included age $(<65,65-74$, $75-84, \geq 85$ ), sex (male, female), race (white, black, Asian, Hispanic, other), number of elixhauser comorbidities ${ }^{31}$ (no comorbidities, $\leq 0,1-5,6-10,11-15, \geq 16$ ), original reason for Medicare entitlement (disabled/ESRD or old 


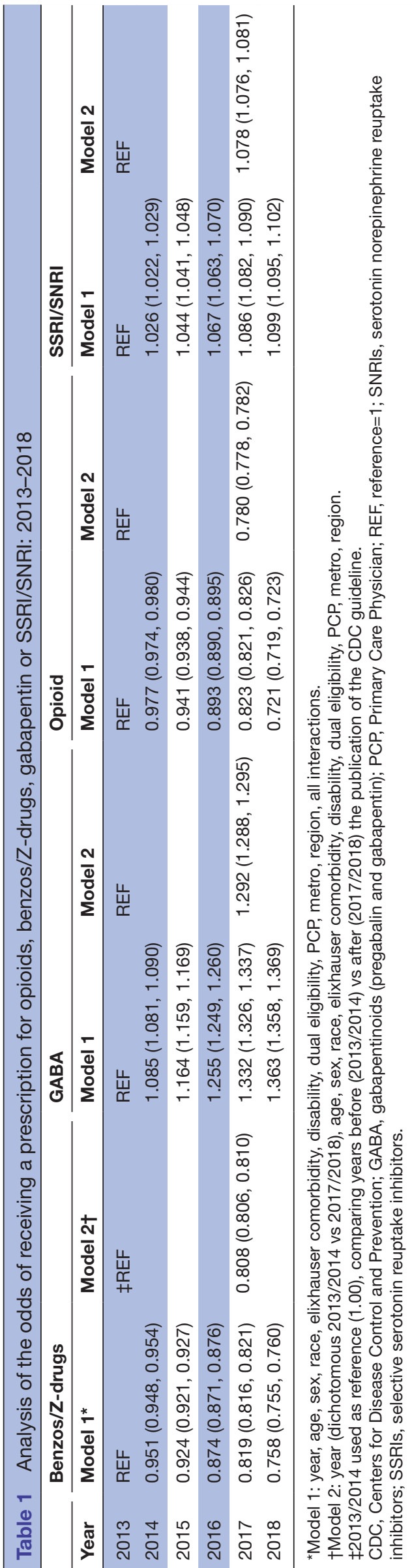

age), Medicaid eligibility (yes, no), metro area (urban, non-metro urban, rural) and US Census division (New England, Middle Atlantic, South Atlantic, East North Central, East South Central, West North Central, West South Central, Mountain, Pacific).

\section{Statistical analyses}

Generalised linear mixed models were used to calculate ORs for opioid use, benzos/Z-drugs use, opioid/benzos/ Z-drugs 30-day use, GABA use and SSRIs/SNRIs use, adjusted for the repeated measure of patient. Two models were used for each of the outcomes. Model 1 calculated the ORs for each year in comparing to 2013 adjusted for all covariates, and model 2 calculated ORs for dichotomous year adjusted for all covariates. All analyses were performed using SAS Enterprise V.7.12.

\section{Patient and public involvement}

The use of a subpopulation of Medicare enrollees allowed us to examine the effectiveness of the various drug combinations on changes in pain and anxiety. Data used in this study were deidentified which prohibited dissemination of results.

\section{RESULTS}

The characteristics of the study cohorts are described in online supplementary eTable2. They contain 4.1-4.3 million enrollees each year from 2013 to 2018; $59.4 \%$ of the enrollees were female; $38.7 \%$ in the 65 to $<75$ age group and $79.6 \%$ were white. While $31 \%$ beneficiaries lacked comorbidities; $12.3 \%$ of the cohort had $15+$ comorbidities. Our sample comprised $27.6 \%$ with disability as original reason for Medicare entitlement and $30.1 \%$ who had Medicaid dual eligibility (see online supplementary eTable 2).

Figure 1 shows the per cent of enrollees who received prescriptions for opioids, benzos/Z-drugs, GABA and SSRIs/SNRIs from 2013 to 2018. The percentage of enrollees who were prescribed opioids decreased from $38 \%$ in 2013 to $30.4 \%$ in 2018 . The percentage receiving benzos/Z-drugs prescriptions decreased from $24.6 \%$ in 2013 to $19.5 \%$ in 2018 . GABA was prescribed in $12.1 \%$ of the study cohort in 2013 and increased to $15 \%$ in 2018. The rate of prescriptions for SSRIs/SNRIs changed little over time, from $23.1 \%$ in 2013 to $23.7 \%$ in 2018.

In table 1 , analysis of data depicting the use of prescribed opioids, benzos/Z-drugs, GABA or SSRIs/ SNRIs. Opioids and benzos/Z-drugs use decreased from 2013 to 2018. In comparing to the use in 2013, GABA use increased by $36.3 \%$ (aOR 1.363; 95\% CI 1.358 to 1.369 ) and SSRIs/SNRIs increased by $9.9 \%$ (aOR 1.099; $95 \%$ CI 1.095 to 1.102$)$ in 2018. Analysis of (2013/2014) vs $(2017 / 2018)$ showed a greater increase in both GABA (aOR, 1.292; 95\% CI 1.288 to 1.295 ) and SSRIs/SNRIs (aOR 1.078; 95\% CI 1.076 to 1.081). Other characteristics associated with each medication were included in online supplemental etable 3 .

Figure 2 presents the time trends in the rates of enrollees for who here was a 30-day combinations of two 


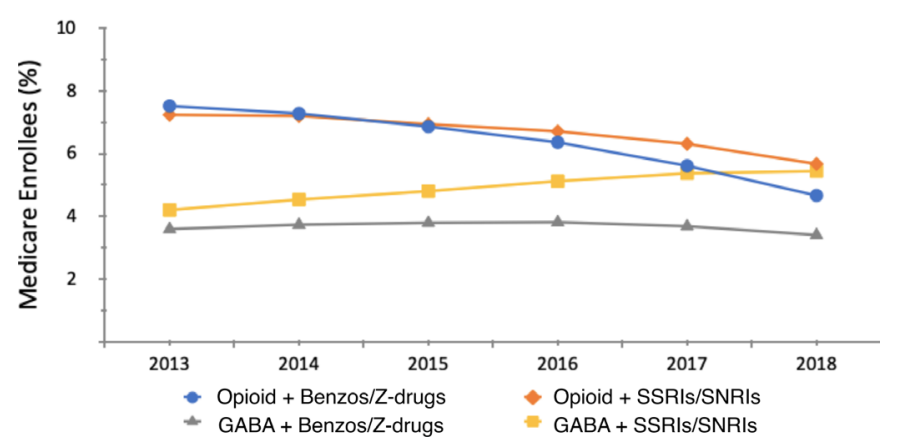

Figure 2 Percentage of Medicare enrollees with a 30day prescription of combined opioid and benzos/Z-drugs, opioid and selective serotonin reuptake inhibitors/serotonin norepinephrine reuptake inhibitors (SSRIs/SNRIs), GABA and benzos/Z-drugs or GABA and SSRIs/SNRIs. Rapid decrease in opioid coprescription with either benzos/Z-drugs or SSRIs/SNRIs and an increase in GABA plus SSRIs/SNRIs coprescriptions is observed from 2013 to 2018. GABA, gabapentinoids (pregabalin and gabapentin).

drugs for 30 days: opioid plus benzos/Z-drugs; opioid plus SSRI/SNRI; GABA plus benzos/Z-drugs; and GABA plus SSRIs/SNRIs. The percentage receiving opioids plus benzos/Z-drugs was $7.5 \%$ in 2013 and declined to $4.7 \%$ in 2018. The percent prescribed both opioid and SSRIs/ SNRIs was $7.4 \%$ in 2013 vs $5.7 \%$ in 2018. The percentage of enrollees concurrently prescribed GABA and benzos/ Z-drugs was relatively unchanged, from $3.6 \%$ in 2013 to $3.4 \%$ in 2018. GABA and SSRIs/SNRIs increased from $4.2 \%$ in 2013 to $5.4 \%$ in 2018.

Table 2 depicts time trends of 30-day use of coprescribed drugs. Coprescribed opioid and benzos/Z-drugs decreased in use by 2018 (aOR 0.626; 95\% CI 0.622 to 0.630 ) comparing to 2013 and the $2017 / 2018$ comparing to $2013 / 2014$ (aOR $0.700 ; 95 \%$ CI 0.697 to 0.703 ). Combined GABA and SSRIs/SNRIs increased in use in 2018 (aOR 1.422; 95\% CI 1.412 to 1.431 ) and in 2017/2018 (aOR $1.335 ; 95 \%$ CI 1.329 to 1.341 ). Other characteristics associated with coprescribing of each combination were included in online supplemental etable 4.

\section{DISCUSSION}

In this retrospective study using a national sample of Medicare beneficiaries data from 2013 to 2018, we found a decrease in the rate of prescribing of opioids and benzos/Z-drugs, and an increase in the rate of gabapentinoid prescribing. Combined opioid and benzos/Zdrugs prescriptions decreased as did combined opioid and SSRIs/SNRIs coprescription, while gabapentinoids and SSRIs/SNRIs coprescription increased. The rate of combined gabapentinoid and benzos/Z-drugs coprescription remained stable throughout the study period.

The time trends in prescribing rates of opioids, benzos/Z-drugs, gabapentinoids and SSRIs/SNRIs alone and in combination before and after the CDC guidelines were uniform across all genders, racial and ethnic groups, and geographic locations. Our findings on the

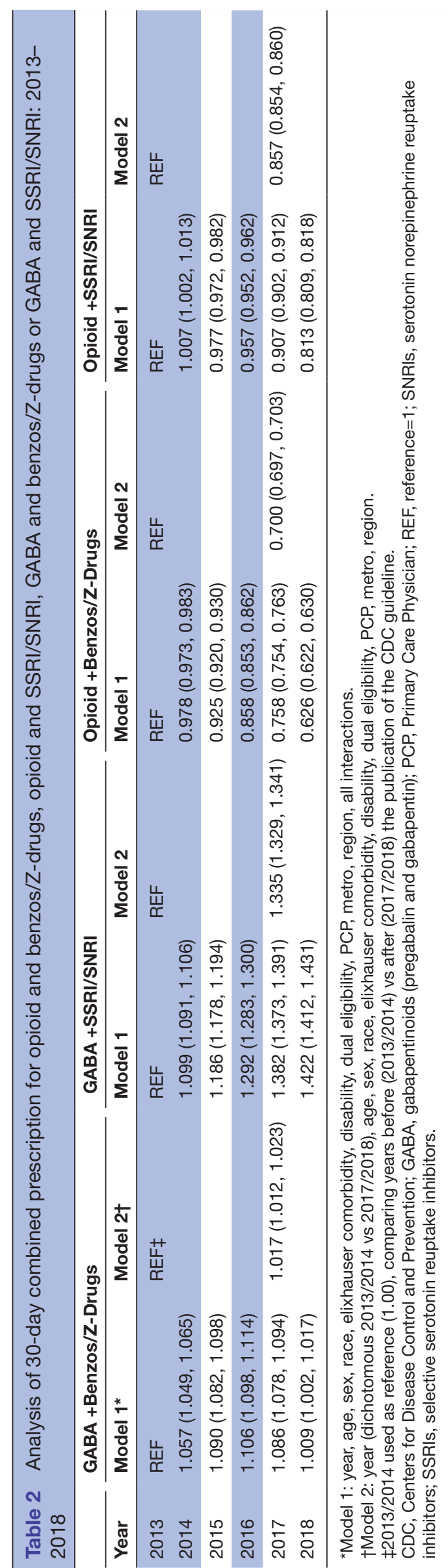


changes in prescribing rates of opioid, benzos/Z-drugs, gabapentinoids and SSRIs/SNRIs, either individually or in combination from 2013 to 2018, suggests a change in prescribers' behaviour. One possible explanation for the change in prescribers' practice could be the potential effects of the widely disseminated 2016 CDC guideline along with subsequent policies by state governments and health payers regulating opioid and benzodiazepine prescribing.

Our study demonstrated a gradual decrease in individually prescribed opioids and benzos/Z-drugs from 2013 to 2016 (figure 1). This early decrease corresponds with the 2013 implementation of the Opioid Safety Initiative, a Veterans Health Administration initiative to reduce high-dose opioids, ${ }^{32}$ and the 2014 DEA hydrocodone rescheduling policy. Our finding showed that the decrease continued in both benzos/Z-drugs and opioid prescribing for another 2 years after the 2016 CDC guidelines that were widely disseminated to all clinicians in the USA. ${ }^{33}$ This finding is consistent with previous studies. ${ }^{81718}$ The pattern of decline in both opioid and benzos/Z-drugs prescribing rates suggests caution on the part of prescribers, considering the well-documented toxicities and risks of both drugs. ${ }^{1-16}$

Individually prescribed gabapentinoid increased by more than $36.3 \%$ during the study period (2013-2018), with a smaller increase observed in SSRIs/SNRIs prescription rates. Our finding suggests that prescribers are using non-opioid (gabapentinoids) and non-benzos/Z-drugs (SSRIs/SNRIs) substitutes as alternatives to treat chronic pain and anxiety. Previous studies have reported on the potential for non-opioid analgesics in decreasing overprescription of opioids for chronic non-cancer pain and in mitigating pre-existing opioid use disorders. ${ }^{25}{ }^{26} \mathrm{We}$ were surprised that we did not observe a more substantial increase in both gabapentinoids and SSRIs/SNRIs prescribing overtime, which suggest insufficient evidence to guide clinicians on the effectiveness and safety of these drugs. Further research is urgently needed to provide data on effects of these drugs on decreasing pain and anxiety, and increasing function and quality of life of patients with chronic pain and comorbid anxiety disorders. More research is also needed on best approaches to managing all the five major types of anxiety disorder-generalised anxiety disorder, obsessive-compulsive disorder, panic disorder and social anxiety disorders-which are common in chronic pain patients and are contributors to excess disability in these patients. ${ }^{34}$

We found that the 30-day co-prescribing of opioids with benzos/Z-drugs decreased from 2013 to 2016, before the publication of the 2016 CDC guidelines, and continued thereafter through 2018, a finding similar to a study by Bohnert et al, based on retail pharmacy data from 2015 to $201^{17}$ but different from Jeffery et al ${ }^{16}$ who described an increase or stabilisation of co-prescribing rates prior to the CDC guidelines. This difference could be due to inherent differences in the study populations observed. Jeffery et $a l^{16}$ included people who were commercially insured or had Medicare Advantage. The difference may also be a reflection of differences in study design. We reported trends from 2013 to 2018 of co-prescribed opioid and benzos/Z-drugs each year while Jeffery $e t \mathrm{al}^{16}$ separately reported trends in the first 90 days in commercially insured beneficiaries. Jeffery $e t a l^{16}$ did, however, find a decrease in trend in the first 90 days in the subset of Medicare Advantage enrollees. Our study findings thus extend decrease in opioid- benzos/Z-drugs coprescribing that occurs in the immediate aftermath of the 2016 CDC guideline to 2 years thereafter with a concomitant increase in prescribing of non-opioid and non- benzos/ Z-drugs alternatives, a direction that is consistent with a recognition of the adverse effects associated with opioids and benzos/Z-drugs coprescribing related accidents, injuries and deaths. ${ }^{11-16} 18$ 35-37

Our finding of increase in the use of combined gabapentinoid and SSRIs/SNRIs from 2013 to 2018 suggest some shift by clinicians to considering alternatives to opioids and benzodiazepines, despite lack of comparative effectiveness and safety data on the use of benzos/Z-drugs and opioid substitutes in the context of chronic pain syndrome and comorbid anxiety disorders; this is clearly an area for future investigations. We used the year 2013 as the reference time point in our analysis of subsequent time points. The odds of receiving a longterm prescription (up to 30 days) of both gabapentin and SSRIs/SNRIs increased in 2018. We also analysed dichotomous years before (2013/2014) and after $(2017 / 2018)$ 2016. The release of the CDC guidelines was associated with an increase in use of substitute drugs, gabapentinoids, and SSRI/SNRIs (adjusted odds ratio (aOR) $1.335 ; 95 \%$ CI 1.329 to 1.341 ) compared with opioid and benzos/Z-drugs (aOR 0.700; 95\% CI 0.697 to 0.703 ). Whether this trend continues beyond 2018 remains to been seen.

There were limitations in our study. First, our study included individuals who filled prescriptions. Using that data, we were unable to verify whether the patients actually took the prescribed drugs, considering that diversion of prescribed opioids and benzos/Z-drugs is a rising concern in the USA. This limitation is generally present in pharmacoepidemiological analyses performed using administrative claims data. Second, these results are only representative of Medicare part $\mathrm{D}$ beneficiaries and not HMO or Medicare Advantage or commercially insured patients. Third, the enrollees under age 65 were highly selected and enrolled because of either disability or endstage renal disease (ESRD) and are thus not representative of the general population under 65 years of age. Fourth, given the large sample size of our study with more than 4 million enrollees per year, even very small differences will be statistically significant, and these may not have substantial clinical significance. We were, therefore, more interested in clinical significance and emphasised results with large effect size. Lastly, the results presented in this study do not assess prescriber factors and outcomes associated with co-prescribing of opioid, benzodiazepine, 
gabapentinoids and SSRI/SNRIs—an area for future investigation.

In summary, we demonstrated an increase in coprescribed gabapentinoids and SSRIs/SNRIs trend across the study period. The increasing use of opioid and benzos/Zdrugs substitutes could reflect a gradual shift in patterns of managing pain and comorbid anxiety disorders, from opioid/benzos/Z-drugs to alternatives that clinicians consider as being safer-despite no high-level evidence. The modest increase in our observed time trends could also reflect the uncertainty in toxicity profile of gabapentinoids and SSRIs/SNRIs when used in combination for a prolonged period-perhaps a reflection of paucity of comparative toxicity data. The long-term toxicity profile of coprescribed gabapentinoids and SSRIs/SNRIs remains to be elucidated. Reduction in the percentage of patients who received prescriptions for benzos/Z-drugs or opioids, either alone or in combination, conveys a potential shift in the prescribing behaviours towards substitute medication for pain and anxiety. It is, however, not clear if these shifts in prescribing behaviour are associated with good pain and anxiety control, fewer toxicities and better quality of life for patients living with chronic pain and comorbid anxiety.

\section{Author affiliations}

${ }^{1}$ Neurology, The University of Texas Medical Branch, Galveston, Texas, USA

${ }^{2}$ Division of Geriatrics and Palliative Medicine \& Sealy Center on Aging, Department of Internal Medicine, The University of Texas Medical Branch, Galveston, Texas, USA ${ }^{3}$ Division of Geriatrics and Palliative Medicine \& Sealy Center on Aging, Department of Internal Medicine, Department of Preventive Medicine and Population Health, Institute for Translational Science, The University of Texas Medical Branch, Galveston, Texas, USA

${ }^{4}$ Office of Biostatistics, The University of Texas Medical Branch, Galveston, Texas, USA

${ }^{5}$ Department of Neurology, Division of Geriatrics and Palliative Medicine \& Sealy Center on Aging, Department of Internal Medicine, Department of Preventive Medicine and Population Health, The University of Texas Medical Branch, Galveston, Texas, USA

Contributors MAR had full access to all of the data in the study and takes responsibility for the integrity of the data and the accuracy of the data analysis. Concept and design: MAR, Y-FK, AE, JSG and JW. Acquisition, analysis or interpretation of data: MAR, Y-FK, AE, JSG and JW. Drafting of the manuscript: MAR, Y-FK, AE, JSG and JW. Critical revision of the manuscript for important intellectual content: MAR, Y-FK, AE, JSG and JW. Statistical analysis: Y-FK and JW. Obtained funding: Y-FK and MAR. Administrative, technical or material support: MAR and Y-FK. Supervision: Y-FK, MAR and JSG.

Funding This work was supported by National Institute on Drug Abuse, National Institute of Health, grant number R01-DA039192.

Competing interests None declared.

Patient consent for publication Not applicable.

Ethics approval The institutional review board of the University of Texas Medical Branch approved this study and waived the need for informed consent because the data were deidentified. IRB\#: 16-0247.

Provenance and peer review Not commissioned; externally peer reviewed.

Data availability statement № data are available.

Supplemental material This content has been supplied by the author(s). It has not been vetted by BMJ Publishing Group Limited (BMJ) and may not have been peer-reviewed. Any opinions or recommendations discussed are solely those of the author(s) and are not endorsed by BMJ. BMJ disclaims all liability and responsibility arising from any reliance placed on the content. Where the content includes any translated material, BMJ does not warrant the accuracy and reliability of the translations (including but not limited to local regulations, clinical guidelines, terminology, drug names and drug dosages), and is not responsible for any error and/or omissions arising from translation and adaptation or otherwise.

Open access This is an open access article distributed in accordance with the Creative Commons Attribution Non Commercial (CC BY-NC 4.0) license, which permits others to distribute, remix, adapt, build upon this work non-commercially, and license their derivative works on different terms, provided the original work is properly cited, appropriate credit is given, any changes made indicated, and the use is non-commercial. See: http://creativecommons.org/licenses/by-nc/4.0/.

ORCID iD

Mukaila A Raji http://orcid.org/0000-0002-7460-7281

\section{REFERENCES}

1 Wilson N, Kariisa M, Seth P, et al. Drug and opioid-involved overdose deaths - United States, 2017-2018. MMWR Morb Mortal Wkly Rep 2020;69:290-7.

2 Jones CM, Lurie PG, Throckmorton DC. Effect of US drug enforcement administration's rescheduling of hydrocodone combination analgesic products on opioid analgesic prescribing. JAMA Intern Med 2016;176:399-02.

3 CDC Guideline for Prescribing Opioids for Chronic Pain. 2016: summary of constituent comments and CDC response. MMWR Morb Mortal Wkly Rep 2016;65:1-49.

4 Dowell D, Haegerich TM, Chou R. CDC guideline for prescribing opioids for chronic pain-United States, 2016. JAMA 2016;315:1624-45.

5 Strickler GK, Kreiner PW, Halpin JF, et al. Opioid Prescribing Behaviors - Prescription Behavior Surveillance System, 11 States, 2010-2016. MMWR Surveill Summ 2020;69:1-14.

6 Guy GP, Zhang K, Bohm MK, et al. Vital signs: changes in opioid prescribing in the United States, 2006-2015. MMWR Morb Mortal Wkly Rep 2017;66:697-04.

7 Zhu W, Chernew ME, Sherry TB, et al. Initial opioid prescriptions among U.S. commercially insured patients, 2012-2017. N Engl J Med 2019;380:1043-52.

8 Olfson M, Wang S, Wall MM, et al. Trends in opioid prescribing and self-reported pain among US adults. Health Aff 2020;39:146-54.

9 Peahl AF, Dalton VK, Montgomery JR. Rates of new persistent opioid use after vaginal or cesarean birth among US Women. [published correction appears in JAMA Netw Open. 2019 Aug 2;2(8):e1911235]. JAMA Netw Open;2019:e197863.

10 Smith BC, Vigotsky AD, Apkarian AV, et al. Temporal factors associated with opioid prescriptions for patients with pain conditions in an urban emergency department. JAMA Netw Open 2020;3:e200802.

11 Baillargeon J, Singh G, Kuo Y-F, et al. Association of opioid and benzodiazepine use with adverse respiratory events in older adults with chronic obstructive pulmonary disease. Ann Am Thorac Soc 2019;16:1245-51.

12 Jones CM, Mack KA, Paulozzi LJ. Pharmaceutical overdose deaths, United States, 2010. JAMA 2013;309:657-9.

13 Sun EC, Dixit A, Humphreys K, et al. Association between concurrent use of prescription opioids and benzodiazepines and overdose: retrospective analysis. BMJ 2017;356:j760.

14 Agarwal SD, Landon BE. Patterns in outpatient benzodiazepine prescribing in the United States. JAMA Netw Open 2019;2:e187399.

15 Hernandez I, He M, Brooks MM, et al. Exposure-Response association between concurrent opioid and benzodiazepine use and risk of Opioid-Related overdose in Medicare Part D beneficiaries. JAMA Netw Open 2018;1:e180919.

16 Jeffery MM, Hooten WM, Jena AB, et al. Rates of physician Coprescribing of opioids and benzodiazepines after the release of the centers for disease control and prevention guidelines in 2016. JAMA Netw Open 2019;2:e198325.

17 Bohnert ASB, Guy GP, Losby JL. Opioid prescribing in the United States before and after the centers for disease control and prevention's 2016 opioid guideline. Ann Intern Med 2018;169:367-75.

18 Wang Y, Delcher C, Li Y, et al. Overlapping prescriptions of opioids, benzodiazepines, and carisoprodol: "Holy Trinity" prescribing in the state of Florida. Drug Alcohol Depend 2019;205:107693.

19 Peckham AM, Evoy KE, Ochs L, et al. Gabapentin for offlabel use: evidence-based or cause for concern? Subst Abuse 2018;12:117822181880131-8. 
20 Pauly NJ, Delcher C, Slavova S, et al. Trends in gabapentin prescribing in a commercially insured U.S. adult population, 20092016. J Manag Care Spec Pharm 2020;26:246-52

21 Goodman CW, Brett AS. Gabapentin and pregabalin for pain - is prescribing a cause for concern? N Engl J Med 2017;377:411-4.

22 Asmundson GJG, Katz J. Understanding the co-occurrence of anxiety disorders and chronic pain: state-of-the-art. Depress Anxiety 2009;26:888-901.

23 McWilliams LA, Cox BJ, Enns MW. Mood and anxiety disorders associated with chronic pain: an examination in a nationally representative sample. Pain 2003;106:127-33.

24 Kascakova N, Furstova J, Hasto J, et al. The Unholy Trinity: childhood trauma, adulthood anxiety, and long-term pain. Int $J$ Environ Res Public Health 2020;17:414.

25 Hooten WM. Chronic pain and mental health disorders: shared neural mechanisms, epidemiology, and treatment. Mayo Clin Proc 2016;91:955-70.

26 Cunningham JL, Craner JR, Evans MM, et al. Benzodiazepine use in patients with chronic pain in an interdisciplinary pain rehabilitation program. J Pain Res 2017;10:311-7.

27 Obata H. Analgesic mechanisms of antidepressants for neuropathic pain. Int J Mol Sci 2017;18:2483.

28 Cadogan CA, Bradley CP, Bennett K. Impact of changes in controlled drugs legislation on benzodiazepine receptor agonist prescribing in Ireland: a repeated cross-sectional study. Eur J Clin Pharmacol 2021;77:903-12.

29 Pottie K, Thompson W, Davies S, et al. Deprescribing benzodiazepine receptor agonists: evidence-based clinical practice guideline. Can Fam Physician 2018;64:339-51.
30 Sharma V, Weir D, Samanani S, et al. Characterisation of concurrent use of prescription opioids and benzodiazepine/Z-drugs in Alberta, Canada: a population-based study. BMJ Open 2019;9:e030858.

31 Elixhauser A, Steiner C, Harris DR, et al. Comorbidity measures for use with administrative data. Med Care 1998;36:8-27.

32 Lin LA, Bohnert ASB, Kerns RD, et al. Impact of the opioid safety initiative on opioid-related prescribing in veterans. Pain 2017;158:833-9.

33 Quality improvement and care coordination: implementing the CDC guideline for prescribing opioids for chronic pain. 2018. National center for injury prevention and control, division of Unintentinal injury prevention. Atlanta, GA Centers for Disease Control and Prevention; 2018. https://www.cdc.gov/opioids/healthcare-admins/pdf/QualityImprovement-Care-Coordination-508.pdf

34 What are the five major types of anxiety disorders? 2014U.S. Department of Health \& Human Services. Available: https://www. hhs.gov/answers/mental-health-and-substance-abuse/what-arethe-five-major-types-of-anxiety-disorders/index.html [Accessed 2021].

35 Nguyen AV, Ross E, Westra J. Opioid utilization in geriatric patients after operation for degenerative spine disease. J Neurosurg Anesthesiol 2020;21:10.1097.

36 Nicol AL, Hurley RW, Benzon HT. Alternatives to opioids in the pharmacologic management of chronic pain syndromes: a narrative review of randomized, controlled, and blinded clinical trials. Anesth Analg 2017;125:1682-3.

37 Gressler LE, Martin BC, Hudson TJ, et al. Relationship between concomitant benzodiazepine-opioid use and adverse outcomes among US veterans. Pain 2018;159:451-9. 